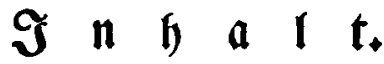

8 Eeite Simngebidte. ..................

1. Die Einngebidte an ton $\mathbf{x} r i r t \ldots \ldots \ldots \ldots \ldots \ldots \ldots$

2. Ebentieferben ..................

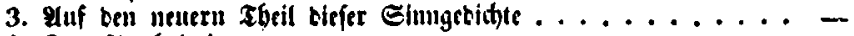

4. Der Etadtetrtim .................... 2

5. Milunber ..................

6. Fn ben gharull ................ -

7. PRerfur unb 2 mor $\ldots \ldots \ldots \ldots \ldots \ldots \ldots \ldots \ldots$

8. Thrar unb Star .................

9. Der geijige Didjter $\ldots \ldots \ldots \ldots \ldots \ldots \ldots \ldots$

10. Inf \&ucinben $\ldots \ldots \ldots \ldots \ldots \ldots \ldots \ldots \ldots \ldots$

11. Fuf bie Eurova ................

12. Pomplls Ranbgut $\ldots \ldots \ldots \ldots \ldots \ldots \ldots \ldots \ldots$

13. \$Biterruf bes Borlgens ...............4 4

14. In ble serren $x$ unb of ..............

15. Die Emigfeit gewiffer Gebidtte . . . . . . . . . -

16. Iuf bas Jungfernftift jul $\ldots \ldots \ldots \ldots \ldots \ldots \ldots$

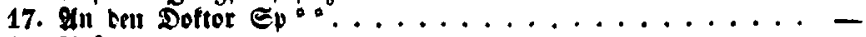

18. श्रuf ben glnemon $\ldots \ldots \ldots \ldots \ldots \ldots \ldots \ldots \ldots$

19. Bavd Gaft .................. 5

20. Fuf ben \&ufub .................

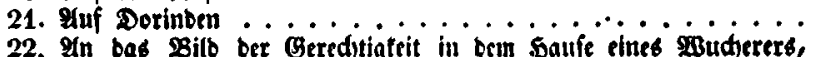

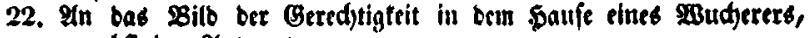
nebit ber entwort ................. -

23. शtuf einen ablid)en Rummfopf ............. -

24. Int sine wúrbige pribatperfou . . . . . . . . . . 6

25. शuf bie Jris ................... -

26. Iuf Frau Irit ................

27. 2luf \&utrin6 Grab .................. -

28. Im Ramen cintr gewiffer spocten, bem ber soünig eine golbene

Dofe fidentte. . . . . . . . . . . . . . - 
29. Fuf ben fillifien Ruf son Migrhis sobe ........... (6

30. शluf ton bargil .................. 7

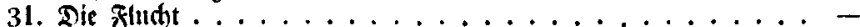

32. Die פूobitbaten .................. -

33. 2n cincu Geijigen .................... -

34. Sing tm fint .................... -

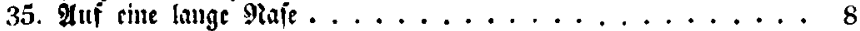

36. श̂ti Etipjen .....................

37. Inif ton Eanttulus ..................

38. In Griflan .......................

39. In ton Enlontent .................. 9

40. 9 tuf ebritenfelben ...................

41. Das bife geib .................... -

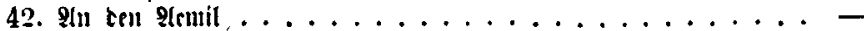

43. Irur an tri $\Xi_{n b i n} \ldots \ldots \ldots \ldots \ldots \ldots \ldots \ldots$

44. Intwort tos Eabin ................. 10

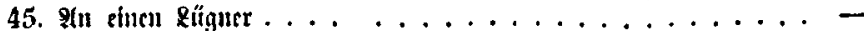

46. Iflf Trill und Trod .................. -

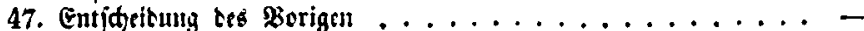

48. In bie $\ldots \ldots \ldots \ldots \ldots \ldots \ldots \ldots$

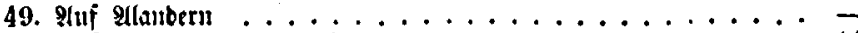

50. Qluf tinen $\mathfrak{B}$ rand

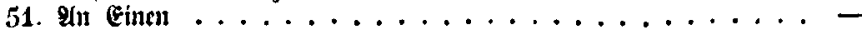

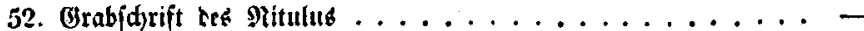

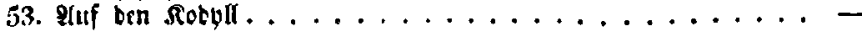

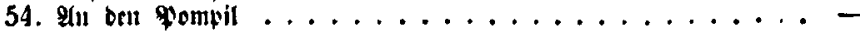

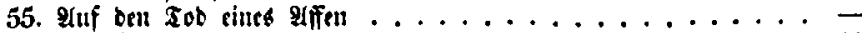

56. Grabjh)rift nuf ebenberifalben ............... 12

57. शuf bie quaji6 ................... -

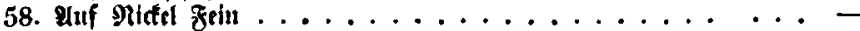

59. \&uf ciute liebbaberiun bos sraucripids............ -

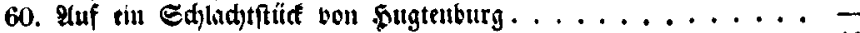

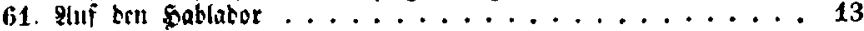

62. Ifuf bol glijon. ....................

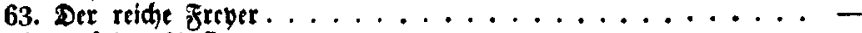

64. शluf bon grufinus .................... -

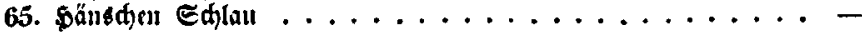

66. Plu bie Dorilis ................... -

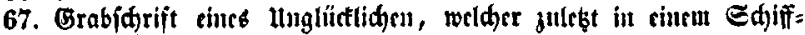

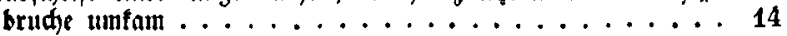

68. In etuen fifledten Maler ................. -

69. 2uf eine gitbjäule tes Ylmor ............... -

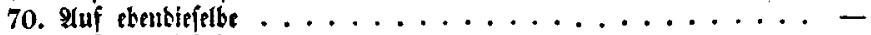

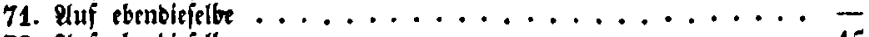

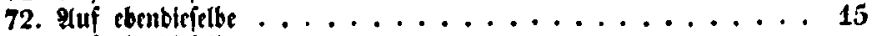

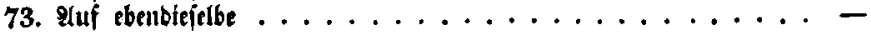

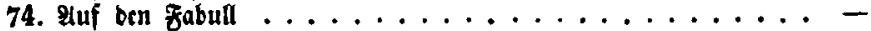

75. In ben trägen $\mathrm{g} \ldots \ldots \ldots \ldots \ldots \ldots$

76. Entidultigung megen unterlaffenes $\mathfrak{B e j u d} 6 . . . . . . . \overline{16}$

77. In ben Panl. .................... 16

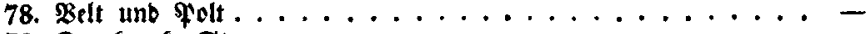

79. Der frante Star ................... -

80. Die blauc \$anb.................. 
81. Dar Eduuftr frauj ................... 17

82. Das Mákdon ..................... -

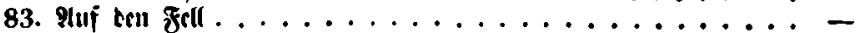

84. In ben berrn $\mathfrak{D}^{*} \ldots \ldots \ldots \ldots$

85. In tinen geigigen 2 ater .................. -

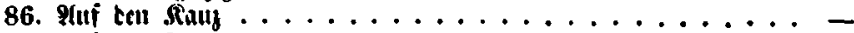

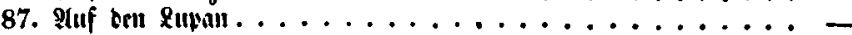

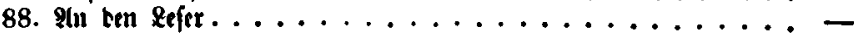

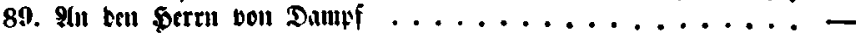

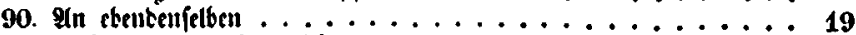

91. Fuf einen gemiffu Dtdfter ..................

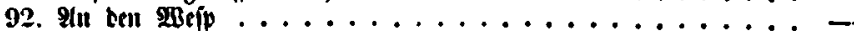

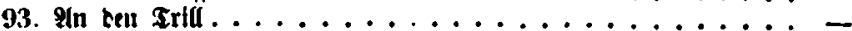

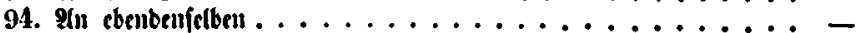

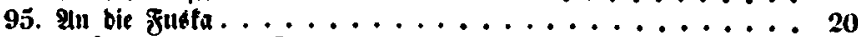

96. Iluf ben Tob ted $\mathfrak{D}$. פncub ................

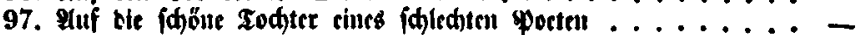

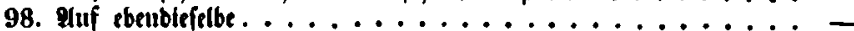

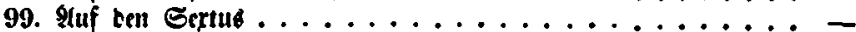

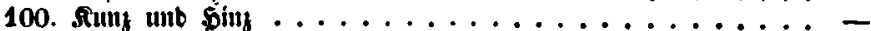

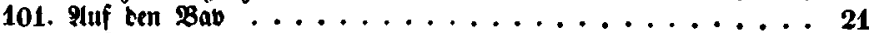

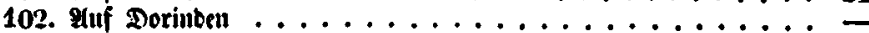

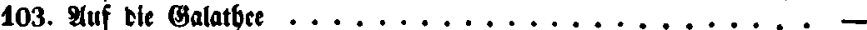

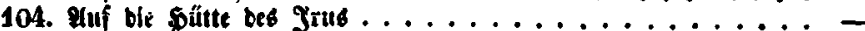

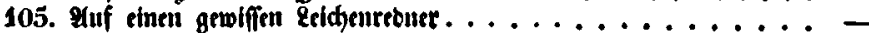

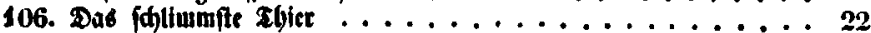

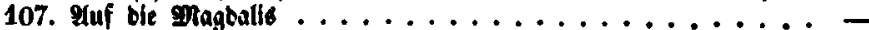

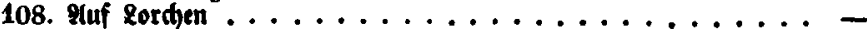

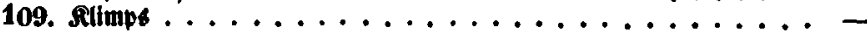

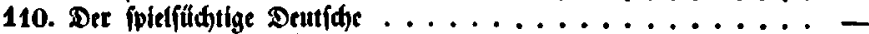

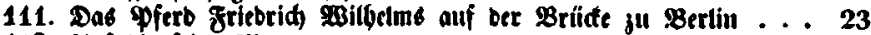

112. Yuf die folge פmumma ..................

113. Cine Gefuntbeit auf bie Gefmbbriten ............ -

114. \&uf einen unnúben getiente" ................ -

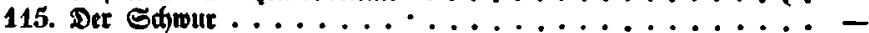

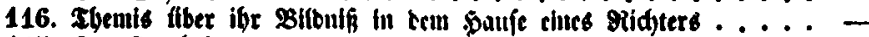

117. Der Furdtfame .................. 24

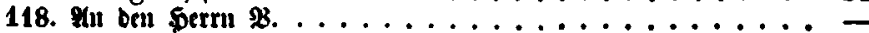

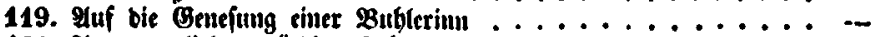

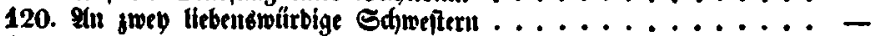

121. Yut beu હiltub ...................... -

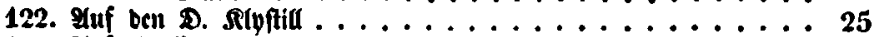

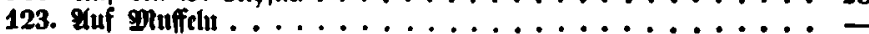

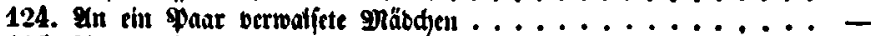

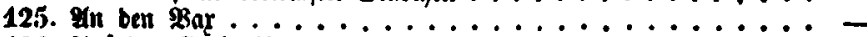

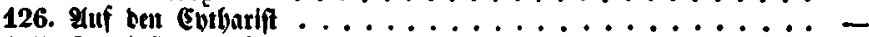

127. Der befte gourf. Fu ciu \$aar Brettipieler ............ -

128. \&uf ben galer Sleft .................. 26

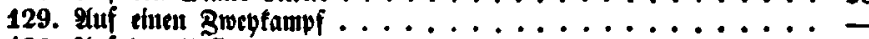

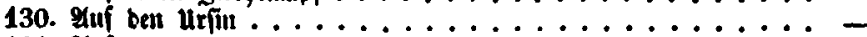

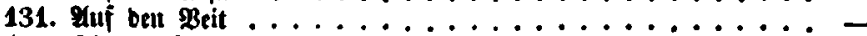

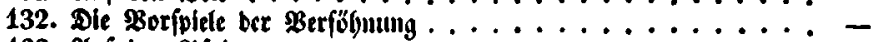

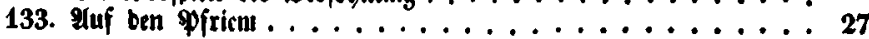




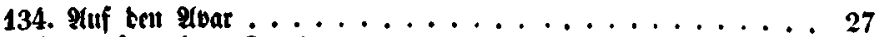

135. Seufzer tines granten .................

136. Iluf ben gaar ...................... -

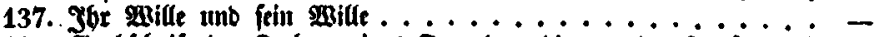

138. Grabjohrlit ber Zoditer cince fretutes, bie bor ber taufe farb -

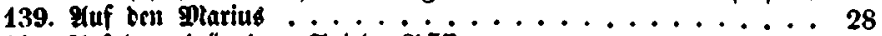

140. Ît ben cinăugigen Epieler \$piff ..............

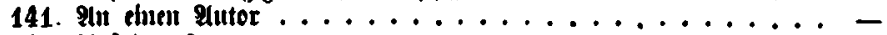

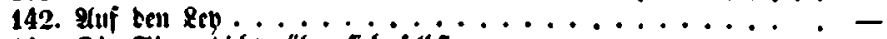

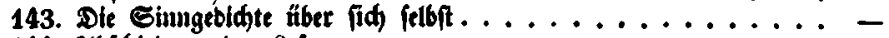

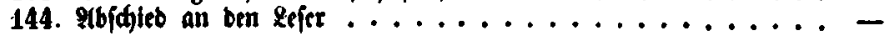

Intyaug, aus gefings edpriften, $1753 \ldots \ldots \ldots \ldots$

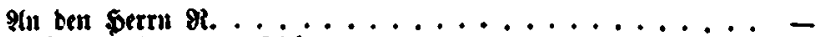

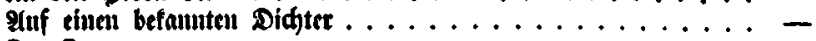

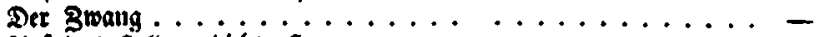

Qtuf bas selbengebiate sermaun. ................

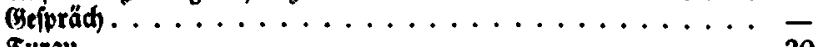

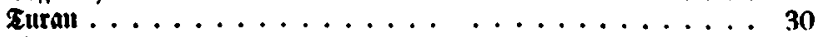

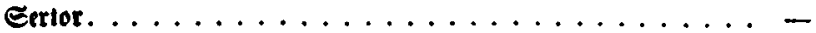

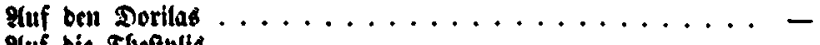

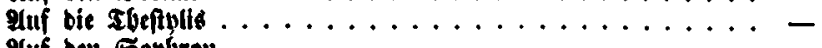

IItf ben Eophrou .................. -

Radjabuung bes 84fien Clungebidts in 3ten Sudje bes Plartials -

Fuf bas Grbtdtt ble Eitutfluth ...............

Yuf ten urbaul ...................... 31

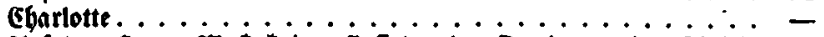

Yluf ben \$erm $2 N$ * ben Erfinter ber Dutabratur tes Birfels -

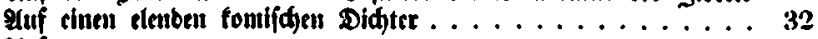

शuf $s=\leq . \ldots \ldots \ldots \ldots \ldots \ldots \ldots \ldots \ldots$

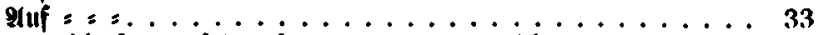

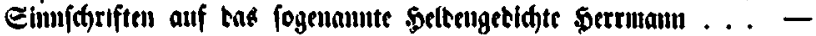

Epigrammata. ................... 35

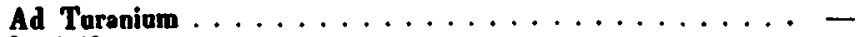

In Ariftum . . .....................

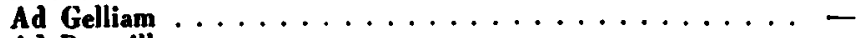

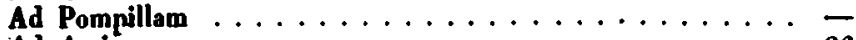

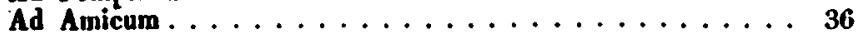

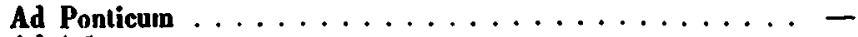

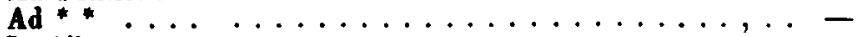

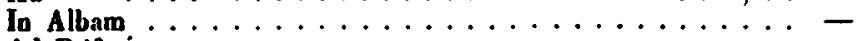

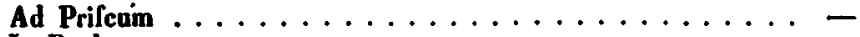

In Paulum ........................

Ad Cacilianum ..................... -

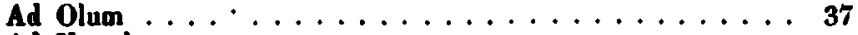

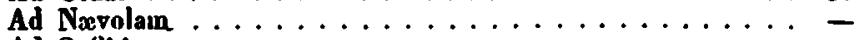

Ad Solibianum ......................

Ad Tuccam ludimagiftrum. ................ -

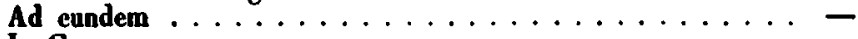

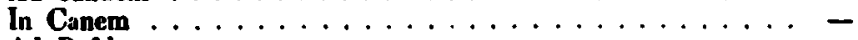

Ad Pofthumum 
Ad Nexcram

In Armilluns

Ad Murlam

\section{[Erftes suth]}

Qin bit Reber

Tic Plamen

Sic siüfr.

Dic Gemifbcil

Die setríbuif. Der Didster unt feiu frcunt.........

Stutwort elted trutufuen Didtuters.............. -

Das aufgebobeuc (Erboi .................. 43

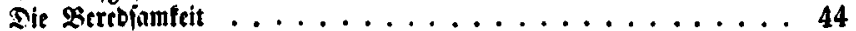

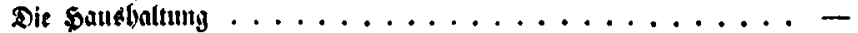

Der Regent ........................

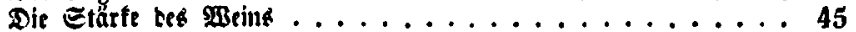

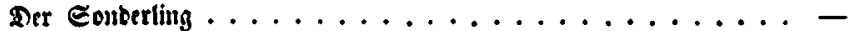

Der alte unt ber junge 2 bein. ................. -

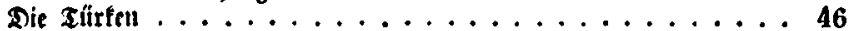

qlteranter ......................

Dic Ed)b̈te bont bintent .................. -

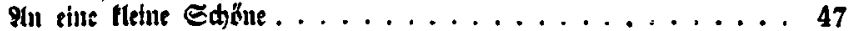

Slad) ber 10. Dbe Plinatrent. ................ -

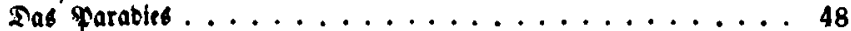

Tie (gefpenfter .................... 49

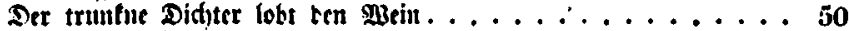

gob ner faulbelt ...................... 51

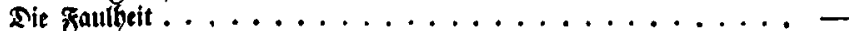

Die Planctenberwobner ...................

Der Gefd)mad ber Yllten .................. 52

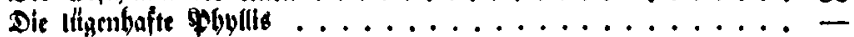

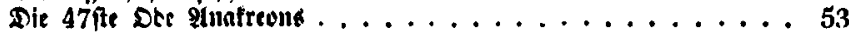

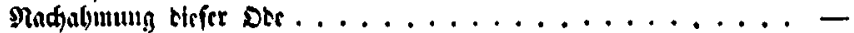

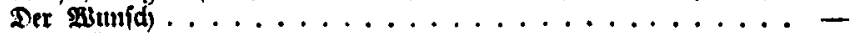

Der gröfte \$2tann .................... 54

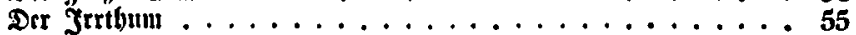

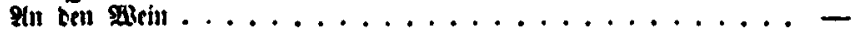

\$ybults an Damon ..................... 56

[Bweutes Bud).]

ffür wou taf) finge.................... -

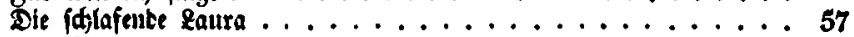

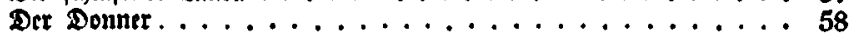

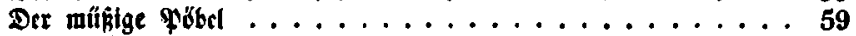

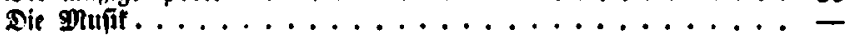

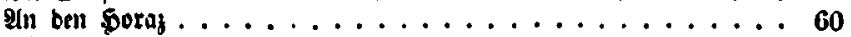

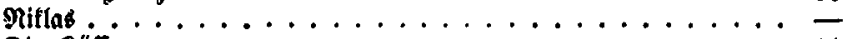

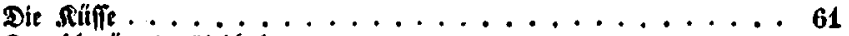

Der fdumörcute qiebbabcr ..................

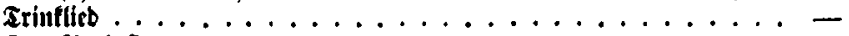

Der 2 erluft . . . . . . . . . . . . . . . 62

Der $\mathfrak{G}_{\text {tnu }} \ldots \ldots \ldots \ldots \ldots$ 
Seite

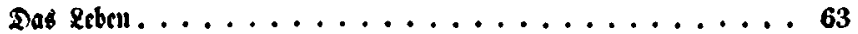

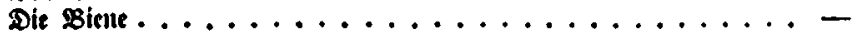

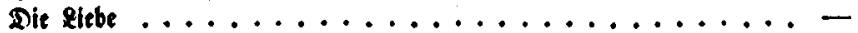

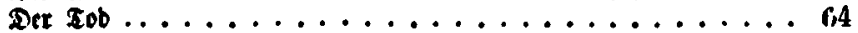

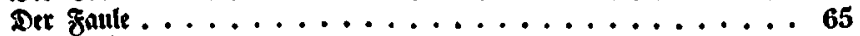

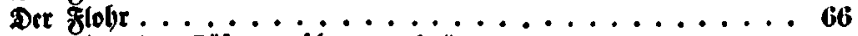

Die wiber ben (Gáfar berídworne gelben ............ -

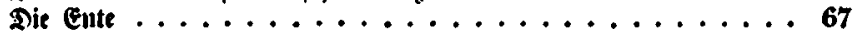

Dle oreb Reidje ber gatut . . . . . . . . . . . . . . 68

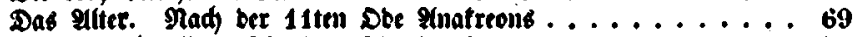

Fin bie Edmalbe. Die 12te Dbe 2luaftront ........... 70

Dle Sunftridter unb ber Didhter ................ - . .

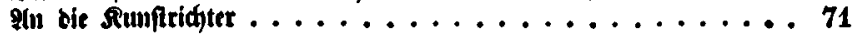

Inbang I. 2Us ben Rleinigfeiten 1751 und aus ben $\mathfrak{E}_{\text {fhrif: }}$ ten 1753 .

Die beríflimmerte Beiten

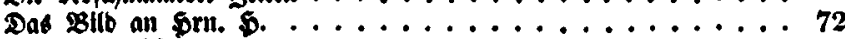

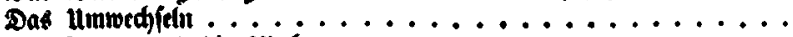

Der Better unb bie פnubme ............... -

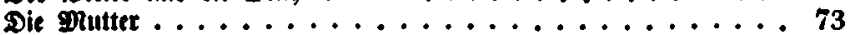

Dit glntmort $\ldots \ldots \ldots \ldots \ldots \ldots \ldots \ldots \ldots \ldots \ldots$

Der Edhlaf .................... 74

Der vbilofophijde stinfer ................

Der Fellirt ................... 75

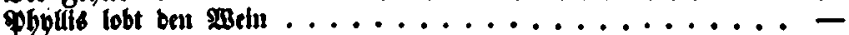

gen ben Etnaftron ....................

Bem to ju gefallen fudje unb nidgt fridje ...........

Das Crobeber1................... 79

Dite Einwobner bet glonbes .............. 80

Der Taufd aut \$r. \$. ................ 81

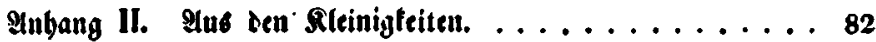

Dle Eparfamfeit $\ldots \ldots \ldots \ldots \ldots \ldots \ldots \ldots \ldots \ldots \ldots$

Die qubedthug ....................

Der beidfeibent \$Bunid)................ 83

Dab Edauferleben ...................

Salomon $\ldots \ldots \ldots \ldots \ldots \ldots \ldots \ldots \ldots$

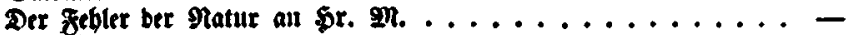

Die fullinmite frau $\ldots \ldots \ldots \ldots \ldots \ldots \ldots \ldots \ldots$

Die Ediffabrt .................. 86

Die sieblidfett . . . . . . . . . . . . . 87

Docn. . . . . . . . . . . . . . . . . 88

J. Der Ciutritt bes 1752 ftu Jabres . . . . . . . . . .

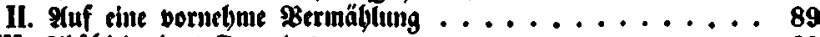

Ill. 2ubidieb etnes freunber ............... 92

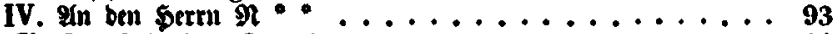

V. Der goo tines greuntes $\ldots \ldots \ldots \ldots \ldots \ldots \ldots \ldots 9$

VI. Der Eintritt bes Jafies 1753 in Berlin ........ 95

VII. Der 24 fie semuer in Berlin $\ldots \ldots \ldots \ldots \ldots \ldots \ldots .97$

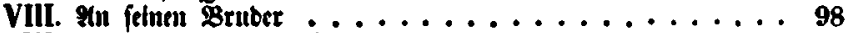

1X. Der Cintritt tes Jabres 1754 in Berlin . . . . . . 99 
Frubch tuto Erjählungen. ............... 101

I. Der Epcrling mo sic gelkmtan ............ -

11. Der gloler unt tir Eule . . . . . . . . . . . . -

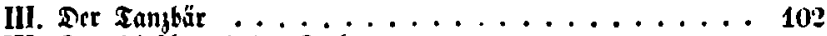

IV. Der Sirfid unt ter fudf $\ldots \ldots \ldots \ldots \ldots \ldots \ldots$.

V. Tie Eonue ................. 103

VI. Das Mufter ber Egen . . . . . . . . . . 105

Dad Gelecimui $\ldots \ldots \ldots \ldots \ldots \ldots \ldots \ldots$

VII. Fantin .................. 107

VIII. Dle cherlide giebe . . . . . . . . . . . . .

X. Der \&üme und bie antifte ............. 109

XI. Das Srucifir .................. 110

XII. Der Eremit . . . . . . . . . . . . . . . 112

XIII. Die Brille $\ldots \ldots \ldots \ldots \ldots \ldots \ldots \ldots \ldots \ldots$

XIV. शị Botenftrom . . . . . . . . . . . . . . 123

Inbang aus bell Crmunterungen jum 2ergnügen bes (ae: mitths

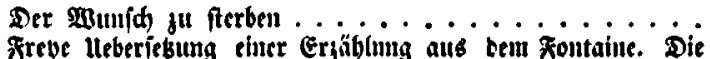

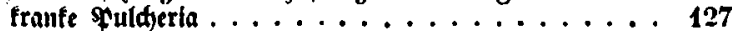

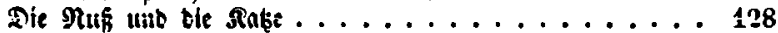

Fitbclut. Drev Bütuer. . . . . . . . . . . . . . 130

Erftes Butch.

1. Dic Eridgeinumg ................. -

2. Der Đamfter unb bic efmejife ............ 131

3. Der göme unt ber waje ............... -

4. Der Ejet unt sas Jagopfero .............. -

5. Jeve ulib wi wierd ............... 132

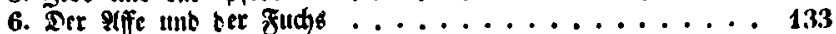

7. Die Nadytignll mo ber qian ............ -

8. Der golf unt ber Edyifitr $\ldots \ldots \ldots \ldots \ldots \ldots \ldots$

9. Das gố unb ber stier . . . . . . . . . . . . 134

10. Die Brtile unb die Radtigall ............ -

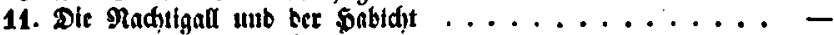

12. Der friegerifide 20 olf . . . . . . . . . . . . -

13. Det phäulr .................. 135

14. Dit Bans . . . . . . . . . . . . . .

15. Dete Eide unb Das Sdbrist ............ 136

16. Dit

17. Die Sperlinge ................. -

18. Der Straulf ................... 137

19. Der Sperling und ter Straur ............ -

20. Dite Sumte $\ldots \ldots \ldots \ldots \ldots \ldots \ldots \ldots \ldots \ldots$

21. Der Fudf und ber Gtord $\ldots \ldots \ldots \ldots \ldots \ldots \ldots$

22. Dit Gute unb ber Edjakgräber . . . . . . . . . . -

23. Die junge Edfmalbe ............... -

24. Sptrops . . . . . . . . . . . . . . . 139

25. Der Pelefau $\ldots \ldots \ldots \ldots \ldots \ldots \ldots \ldots \ldots$. . . . .

26. Der Qüwe unb ter Tieger. . . . . . . . . . 140 
27. Der Ctitr unt ber \$irid) ............... 140

28. Der Ejel unb ter \$olf ................. -

29. Der Epringer int Edjadje ................ 141

30. Qlefoptts unb ter Cfal

\section{Sిmeyted Buth.}

1. Die eberne Bitbjäule

2. Sertules

3. Der Snabe und bie edlange .............

4. Der \$olf auf brm aobtbette $\ldots \ldots \ldots \ldots \ldots \ldots$ 143

5. Der Stler unb bas Salb

6. Die Pfauen unb die Sräbe ............. 144

7. Der Q'́de mit beun Efel

8. Der Efel mit temi Qüme"

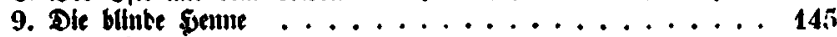

10. Die Efel

12. Jupiter umb gpollo $\ldots \ldots \ldots \ldots \ldots \ldots \ldots \ldots \ldots$

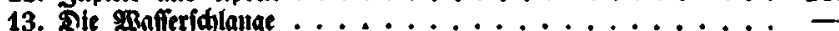

14. Der fouds unt bie karbe .............. -

15. Der Gabe unb ver fucts ............. 148

16. Der Geritige ...................

17. Der Rabe ................... 149

18. 3cbs unb bas edaf

20. Der פMann unt ber Sunb $\ldots \ldots \ldots \ldots \ldots \ldots \ldots$

21. Die Traube ..................

22. Der fudb $\ldots \ldots \ldots \ldots \ldots \ldots \ldots \ldots \ldots \ldots \ldots \ldots$

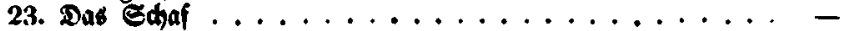

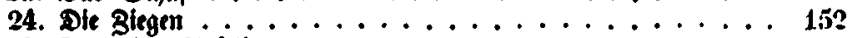

25. Der wilbe Ipfelbaum ............... -

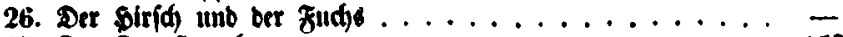

27. Der Dornftratud ................ 15.3

28. Die gurien ...................

29. Jireflas ..................

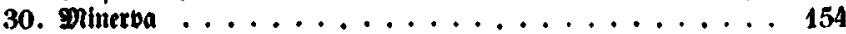

Drittes $\mathfrak{B u d}$.

1. Der Befitger bes Bogens $\ldots \ldots \ldots \ldots \ldots \ldots \ldots \ldots$ 15is

3. Der Geif bes Salonto ................ -

4. Dad Gefident ber frenen ................ -

5. Das Sajaf und bie Sdjwalbe ............. 150

6. Der Rabe ....................

7-10. Der Pengitteit ber ablere ........... 157

11. Der Bär unb ber Elephaut $\ldots \ldots \ldots \ldots \ldots \ldots \ldots \ldots$

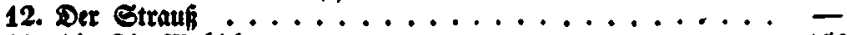

13. 14. Die \$Boblthaten $\ldots \ldots \ldots \ldots \ldots \ldots \ldots$. . . . . . .

15. Die Cidje ................... -

16-22. Die cepdidte bes alten \$olfo . . . . . . . . -

23. Die Praus ................... 163

24. Die Edhwalbe ..................

25. Der $\mathscr{\text { Qbler }} \ldots \ldots \ldots \ldots \ldots \ldots \ldots \ldots \ldots$ 
26. Der juuge unb tor alte \$iríd) .............. 164

27. Ser Pfiul unb tor Shatu

28. Der Sirid $\ldots \ldots \ldots \ldots 165$

29. Rer glter nut ter fitth .............. -

30. Der ๔düfer mub tic Nad)tigall ... . . . . . . . . . -

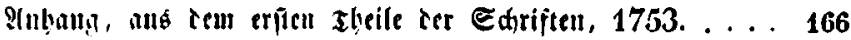

Der Riefe.

Fer Falfe.

Damon tumo sbrotor

Fragumentc.

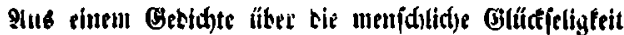

Plis einen (Gebidte an ben Serrn Baron bou $\mathcal{E}_{p}$

Ylus einen Bebldte über ben jeşigen Bridumact fit ber Poefie . . 173

Ylü einem Gebidte an ten Serru $\mathfrak{M}^{*} * \ldots \ldots \ldots 174$

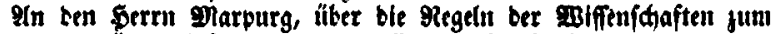

Berguifycu; befonters ber Pocfie unb tontunf .......

Tie Pleltgion. Erfter Grfang ...............

(ijedidte, fo man nad friuem $\mathfrak{T o b e}$ unter feinen Papieren gefunden, und savon einige fđon in Dem Göttingifden Shilıenalinaitadie geftanben.

Fuf Rabeners Tob, als nah weldjem exft feiue übrigen Shiff: ten all bas gidht fommen follten .............. 2uf ben Strit bes Serrn 20 fens mit ben \$ittenbergtidjen Theologen

Sic grofie sidelt

Hutet tas sotlonis bes sönig von Preuket

Doppelter gtutsen einer zraut

Puben eines fermen Cartelt

Der 2 linbe

Ruf th Earuffer

Set Qrme

Sum tutb Sluy

शiı einen Eedsklgjäbrigen

In ben Dímm

Elarum id) wieber Epigramme madie

lleber bas Billonif eilues greuntes

Jil ein Stammbuh), in wridsem the bereits sierforbenen mit ed=

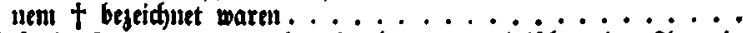

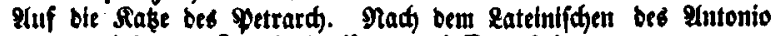
Duerci it bent Ioferiptionibus agri Patavini

Grabidyrift auf $\$ B$ oltatren.

Dle serleumbung

Yu tin Stammbud)

Robipruch bes finisuen (Geid)lechis

शlls ber Serjog Ferbinant bie Rolle bes igameninone, bes enfen

Felbberm ber Griechen, fpielte.

Int ciute Edjaupielers Etanmbud

3in ciu Stammbud)

Qieb alls bom Eranijam 


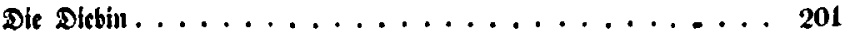

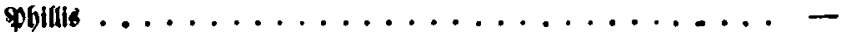

gacd)us unb \$clcua. ................... -

In İmor ........................ 202

Srltenlteb ber Spartaner .................. -

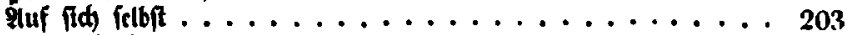

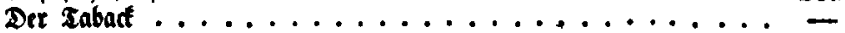

Relutatio Papatus ................... 204

Der nete golibau ................... 205

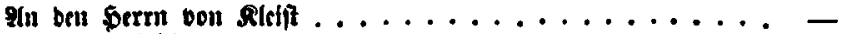

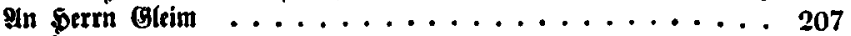

Drpbens ......................... -

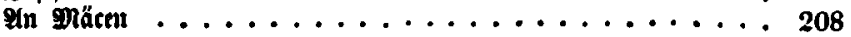

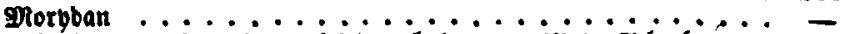

Sdjlufrebe fu ehem Sraueripiele, gebalten bon פNab. Squud . . . 200

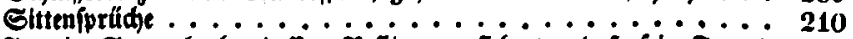

In ein Stammbud), beffen Befiker berfidjerte, baß fein Zreunb

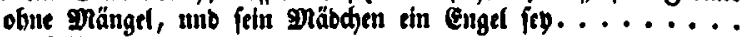

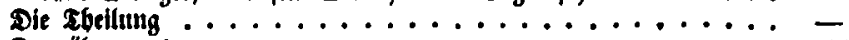

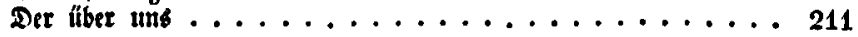

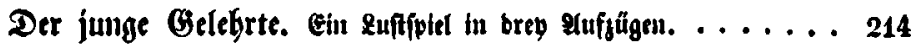

Dic Sube11. Ein \&uftipiel in einem Iufzuge. . . . . . . . 304

Der Mifogyn. Cin euftptel in brey elufyügen. . . . . . . 341

Der Frengeift. Ein Quftpiel in fünf $\mathcal{Y} u f$ gügen. . . . . . . 387

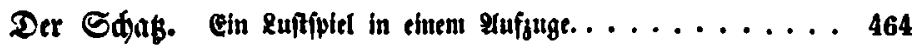

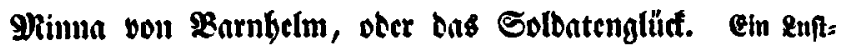

fplet his fünf eluffilgen. . . . . . . . . . . . . . 509 